\title{
The Dynamics of Two Diffusively Coupled Predator-Prey Populations
}

\author{
Vincent A. A. Jansen \\ School of Biological Sciences, Royal Holloway, University of London, \\ Egham, Surrey TW20 0EX, United Kingdom \\ E-mail: vincent.jansen@rhbnc.ac.uk
}

Received December 15, 1999

\begin{abstract}
I analyze the dynamics of predator and prey populations living in two patches. Within a patch the prey grow logistically and the predators have a Holling type II functional response. The two patches are coupled through predator migration. The system can be interpreted as a simple predator-prey metapopulation or as a spatially explicit predator-prey system. Asynchronous local dynamics are presumed by metapopulation theory. The main question I address is when synchronous and when asynchronous dynamics arise. Contrary to biological intuition, for very small migration rates the oscillations always synchronize. For intermediate migration rates the synchronous oscillations are unstable and I found periodic, quasi-periodic, and intermittently chaotic attractors with asynchronous dynamics. For large predator migration rates, attractors in the form of equilibria or limit cycles exist in which one of the patches contains no prey. The dynamical behavior of the system is described using bifurcation diagrams. The model shows that spatial predator-prey populations can be regulated through the interplay of local dynamics and migration. (c) 2001 Academic Press

Key Words: diffusive instability; dispersal; paradox of enrichment; prey-predator cycles; Moran effect.
\end{abstract}

\section{INTRODUCTION}

Well-mixed ecological systems can have very different dynamics from systems in which the movement of individuals is limited (Crowley, 1978; Hassell et al., 1991; De Roos et al., 1991; Adler, 1993; Holyoak and Lawler, 1996a,b; Rohani et al., 1997). In well-mixed systems the dynamics quickly become synchronized, in the sense that the dynamics will be the same at different locations. If movement is limited this is not the case and spatial patterns develop. The spatial averages in well-mixed systems can differ largely form their counterparts with limited movement, due to the asynchronous dynamics that can occur in the latter. In most cases asynchronous dynamics lead to the increased persistence of populations (Hassell et al., 1991; De Roos et al., 1991; Allen et al., 1993; Adler, 1993; Jansen, 1995a).
Oscillations in densities have been observed in predator-prey, host-parasite, and host-pathogen populations. Simple models of such systems with well-mixed populations also show vigorous oscillations that can bring the populations to the brink of extinction or beyond. The oscillations observed in many data series of natural predator-prey populations are normally not as vigorous as the fluctuations predicted by mathematical models. In contrast, populations of predators and prey that are kept in laboratory environments, where the amount of space available is much smaller than in a natural population, often do show oscillations which drive the populations to extinction (Gause, 1934; Huffaker, 1958; Maly, 1969). This suggests that spatial interactions are an important element in the regulation of populations which have a tendency to oscillate in isolation. 
A simple formalism to describe spatially interacting populations is provided by metapopulation models. A metapopulation consists of a set of local populations, living in patches, that are coupled through migration (for reviews see Gilpin and Hanski (1991), Hastings and Harrison (1994), and Hanski and Gilpin (1997)). A metapopulation persists when local populations go through cycles of colonization and subsequent extinction, as long as these cycles proceed asynchronously (Taylor, 1990). In a predator-prey metapopulation it is not difficult to imagine that the local populations can go through large fluctuations, perhaps actually going extinct, but that at the metapopulation level these cycles are damped when the local dynamics proceed out of phase.

In itself this may sound like a truism: when local dynamics proceed out of phase the effects of local fluctuations will simply average out in the regional densities. However, this argument hinges on the asynchrony of the fluctuations in the local densities. The crucial question thus is whether asynchronous fluctuations in the local densities are to be expected in metapopulations. Migration couples local populations in a metapopulation (Hanski, 1991) but it also tends to reduce differences between local populations (Murray, 1993; Solé and Gamarra, 1998; Jansen, 1999). When no differences between the local populations exist, the local patches proceed in phase and are phase locked. If the dynamics are not phase locked some mechanism must be at work that counteracts the synchronizing effect of diffusion.

There is empirical evidence that asynchronous local dynamics occur in metapopulations (Nachman, 1991; Van de Klashorst et al., 1992; Holyoak and Lawler, 1996a,b; Janssen et al., 1997). Asynchronous local dynamics have also been demonstrated in many simulation studies (e.g., Hassell et al., 1991; Comins et al., 1992, Rohani et al., 1997) and in simple analytic models for a single species metapopulation (Gyllenberg et al., 1993; Hastings, 1993). Despite this there is no complete understanding of how and when asynchronous local dynamics may appear.

There are also well-documented examples of populations with a patchy structure in which the dynamics are highly synchronized (Grenfell et al., 1998; Koenig, 1999, and references therein). The explanation that is predominantly used for synchronized population dynamics is the synchronizing force exerted by correlated environmental noise. This mechanism, which has become known as the Moran effect, works well for populations in which the density-dependent feedback is linear (Royama, 1992; Koenig, 1999; Hudson and Cattadori, 1999; Ripa, 2000).
For oscillating populations the density dependence is often highly nonlinear, in which case it is not clear whether the Moran effect works (but see Ripa (2000)). There has been some debate about whether phase locking can offer an alternative explanation for synchronized population dynamics (Jansen, 1999; Ranta et al., 1999).

In this paper I will study the dynamics of coupled oscillating population in the absence of environmental noise. One of the aims is to establish under what conditions synchronous or asynchronous oscillations naturally arise. It is known from the theory of coupled oscillators (Winfree, 1980; Ashwin and Swift, 1992; Murray, 1993; Solé and Gamarra 1998; Lloyd and May, 1999) that synchronized oscillations through phase locking are a generic phenomenon. I will therefore concentrate on the occurrence of asynchronous dynamics and the conditions under which these arise from the interplay of the local population dynamics and the spatial interactions between locally oscillating populations.

The metapopulation that is used here is very simple: a system of two identical patches in which the dynamics are coupled through the migration of individuals. The work in this paper builds on work on two diffusively coupled identical patches in which the dynamics in each patch were given by the Lotka-Volterra predator-prey equations without self limitation (Jansen, 1994, 1995a; Czaran, 1998; Jansen and De Roos, 2000; Jansen and Lloyd, 2000). This model has a family of closed orbits on which the dynamics are synchronized. By studying the stability of these orbits it was shown that for appropriate choices of parameters synchronized oscillations with a large amplitude can be unstable. Orbits leading away from these unstable closed orbits converge toward synchronized oscillations with a small amplitude, hence the population dynamics in the long run always become synchronized. Here I will show that in a two-patch metapopulation, where the local population dynamics are described by a predator-prey system with logistic prey growth and a Holling type II functional response, limit cycles with synchronous oscillations can be unstable, and that attractors with asynchronous dynamics exist. Although the model studied in this paper is for a predator-prey system, host-parasite or host-pathogen system qualitatively similar results are to be expected.

The model studied here deserves attention not only for its biological significance but also for its surprisingly rich dynamical behavior. My simulations showed that, for parameters under which a well-mixed model exhibits sustained synchronous oscillations, the two-patch model can exhibit different types of asynchronous periodic behavior. In addition I found quasi-periodic and intermittent 
chaotic attractors, in all of which the local dynamics proceed out of phase. To go beyond simulations results I performed a numerical bifurcation analysis of this model. By means of bifurcation diagrams the genesis of the attractors is described. Such an analysis has the advantage over simulation methods in that it offers a structured way to find the possible attractors.

I show that through the interplay of local dynamics and migration both synchronous and asynchronous oscillations arise and that the fluctuations in the total densities are damped compared to the fluctuations in a single isolate population. These results have a wider significance: the one but crudest discretization of space is a subdivision in two regions. The model used here can easily be extended to accommodate more patches and offers a natural starting point for the investigation of spatial systems (Jansen and Lloyd, 2000).

\section{MODEL DESCRIPTION}

I describe the local interaction between predator and prey by the Rosenzweig-MacArthur (1963) model in which the prey population grows logistically and the predator has a Holling type II functional response:

$$
\begin{aligned}
& \frac{d N}{d t}=r N\left(1-\frac{N}{K}\right)-\frac{b N P}{b+N} \\
& \frac{d P}{d t}=\frac{b N P}{b+N}-\mu P .
\end{aligned}
$$

The variable $N$ denotes the density of the prey population and $P$ the density of the predator population. The variables in (1) have been scaled to reduce the number of parameters. The remaining parameters have the following interpretation: $r$, prey growth rate at low prey densities; $K$, carrying capacity of the prey population; $b$, the saturation value of the functional response; $\mu$, the predator death rate in the absence of prey. The scaling chosen preserves the carrying capacity, which is the predominant regulating factor, as a bifurcation parameter. Furthermore, by taking the limit of $K$ and $b$ tending to infinity, system (1) reduces to the Lotka-Volterra system without self limitation. System (1) describes the population dynamics of a well-mixed predator and prey population. The dynamics are well known: system (1) allows for a single nontrivial two-species equilibrium which can be either locally stable or unstable. In the latter case a stable limit cycle exists (see, e.g., Murray, 1993).
Next consider a population consisting of two subpopulations, inhabiting two patches which are coupled by migration. I have assumed that the populations within a patch are well mixed and that the dynamics are given by (1). The two-patch metapopulation is described by

$$
\begin{array}{r}
\frac{d N_{i}}{d t}=r N_{i}\left(1-\frac{N_{i}}{K}\right)-\frac{b N_{i} P_{i}}{b+N_{i}} \\
\frac{d P_{i}}{d t}=\frac{b N_{i} P_{i}}{b+N_{i}}-\mu P_{i}+d\left(P_{j}-P_{i}\right) \\
\quad i, j \in\{1,2\} ; \quad i \neq j,
\end{array}
$$

where $N_{i}$ and $P_{i}$ denote the densities of the prey and predator population in patch $i$. The parameter $d$ denotes the per capita predator migration rate. I assumed that prey do not migrate.

Because the model parameters were chosen the same in both patches, the model describes a homogeneous environment. This makes the model symmetrical in the sense that if a solution of system (2) is known, a second solution can be constructed by swapping the subscripts in the population densities. This can be expressed more formally by defining the reflection operator, $R$, as

$$
R(x, y, u, v)=(u, v, x, y)
$$

If $S(t)=\left(N_{1}(t), P_{1}(t), N_{2}(t), P_{2}(t)\right)$ is a solution of (2), $R S(t)$ is also a solution. Note that by applying the reflection operator twice one regains the original solution, hence the system is $\mathbb{Z}_{2}$-equivariant (Kuznetsov, 1995). Moreover, the diagonal subspace $D=\left\{\left(N_{1}, P_{1}, N_{2}, P_{2}\right)\right.$ $\left.\in \mathbb{R}_{+}^{4} \mid N_{1}=N_{2}, P_{1}=P_{2}\right\}$ is the fixed-point subspace in the sense that every point $x$ in $D$ is its own $R$ image: $R x=x$. For any solution in the subspace $D$ the densities in both patches are equal; therefore migration has no effect and hence $D$ is invariant under (2). Moreover, on $D$ the dynamics of $N_{i}$ and $P_{i}$ are given by (1). Hence, when the parameters are chosen such that for (1) a limit cycle exists, system (2) also has limit cycle on the diagonal $D$, denoted by $\Gamma(t)$. This limit cycle $\Gamma$ is fixed under $R$ because $R \Gamma(t)=\Gamma(t)$. Such cycles are called $\mathrm{F}$-cycles in the terminology of $\mathbb{Z}_{2}$-equivariant systems (Kuznetsov, 1995). On this F-cycle the densities in the two patches are equal at all times and the oscillations in the local densities are strictly in phase. In order to locate asynchronous attractors of (2), I studied the stability of the equilibria and the F-cycle $\Gamma$ and continued the $\omega$-limit sets that bifurcate off these solutions. (An $\omega$-limit set is the set of accumulation points of an orbit (Hofbauer and Sigmund, 1998). Such sets, which can be equilibria, 
limit cycles or more complicated sets, need not be attractors but all attractors are $\omega$-limit sets.) A possible way of locating attractors is by continuing $\omega$-limit sets under a change of parameters.

Guided by the observations on two coupled patches with Lotka-Volterra local dynamics (Jansen, 1994, 1995a) the other parameters were fixed at $\mu=r=1$, $b=9.96$. This particular value for $b$ was chosen for numerical convenience; for other values of $b$ qualitatively similar results can be obtained. For the parameter values chosen, all equilibria of (1) are unstable and the twospecies equilibrium is surrounded by a stable limit cycle. As bifurcation parameter I used $d$, which expresses the coupling between the two patches and $K$, which represents the carrying capacity. For location and continuation of equilibria and limit cycles the program LOCBIF (Khibnik et al., 1993) has been used.

\section{LOCAL BIFURCATION STRUCTURE}

The bifurcation structure of (2) is rather complex. I therefore first describe the equilibria and the behavior of the decoupled dynamics $(d=0)$ followed by the dynamics for small migration rates. The bifurcation structure for larger predator migration rates is explained, starting from the bifurcation structure within the face $N_{2}=0$, i.e., when no prey is present in patch 2 . Because the prey migration rate is zero, this subspace is invariant and forms a convenient starting point to investigate the complete state space $\mathbb{R}_{+}^{4}$. In two separate subsections the connections between $\omega$-limit sets in $\mathbb{R}_{+}^{4}$ and the faces $N_{i}=0$ are described.

\subsection{Equilibria}

System (2) maximally allows for nine equilibria (Table 1). The equilibria are denoted by $E_{i j}$, where $i$ refers to the number of species in patch 1 and $j$ to the number of species in patch 2 . When more equilibria are possible for a certain combination a letter added to the subscript indicates this.

The equilibria $E_{00}, E_{11}$, and $E_{22 a}$ all lie in $D$ and correspond to the equilibria of (1) with identical equilibrium densities in both patches. Equilibrium $E_{00}$ is always a saddle; $E_{11}$ is stable for $K<1$ and a saddle otherwise. Equilibrium $E_{22 a}$ is positive if and only if $K>\frac{b \mu}{b-\mu}$. It goes through a Hopf bifurcation for $K=$ $b \frac{b+\mu}{b-\mu}$ and is locally stable if and only if $K \leqslant b \frac{b+\mu}{b-\mu}$ and unstable and surrounded by the limit cycle $\Gamma$ if $K>b \frac{b+\mu}{b-\mu}$.
TABLE 1

\section{The Nine Equilibria of (2)}

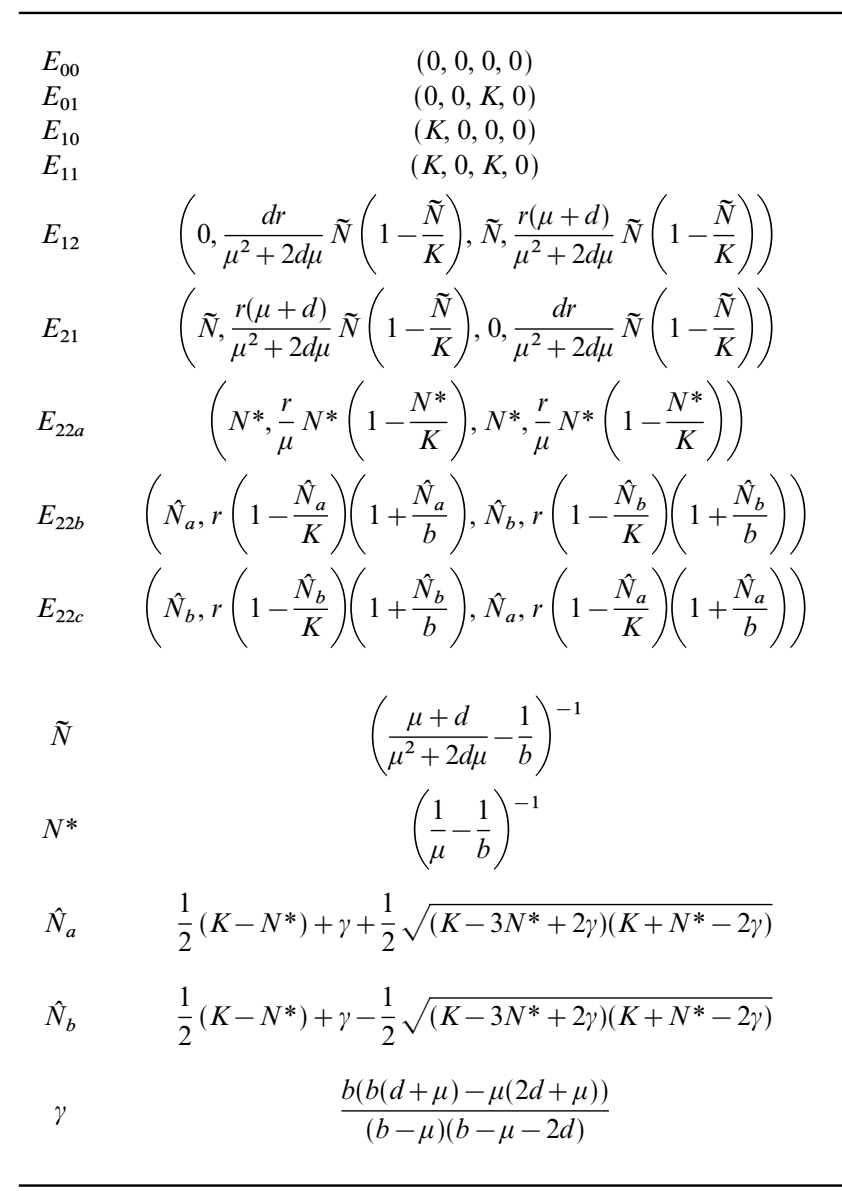

Note. For computation of the equilibria see Jansen (1994).

Four equilibria are possible in which one of the patches contains no prey $\left(E_{01}, E_{10}, E_{21}\right.$ and $\left.E_{12}\right)$. These equilibria lie within one of the invariant subspaces $\left\{\left(N_{1}\right.\right.$, $\left.\left.P_{1}, N_{2}, P_{2}\right) \in \mathbb{R}_{+}^{4} \mid N_{i}=0, i=1,2\right\}$. In what follows I will refer to these subspaces as the faces $N_{i}=0$. These four equilibria form two reflection symmetrical pairs: $R E_{01}=$ $E_{10}$ and $R E_{21}=E_{12}$.

Furthermore, a reflection symmetrical pair of equilibria, $E_{22 b}$ and $E_{22 c}$, can exist in the positive state space. These equilibria branch off the equilibrium $E_{22 a}$ in a pitchfork bifurcation. It is well known that the equivalent of $E_{22 a}$ in a reaction diffusion system in which the local dynamics are given by (1) can only lose its stability in a Hopf bifurcation (Segel and Jackson, 1972). However, once $E_{22 a}$ has lost its stability it can undergo a pitchfork bifurcation in which $E_{22 b}$ and $E_{22 c}$ branch off. 


\subsection{Decoupled Patches}

When $d=0$ the patches are decoupled and the dynamics in both patches are described by (1). For the parameters chosen, system (1) allows a limit cycle and three unstable equilibria with zero, one, or two species present. The $\omega$-limit sets of the system of two patches consist of all possible combinations of these $\omega$-limit sets.

The decoupled system has nine equilibria which consist of combinations of equilibria of (1). The densities in the five equilibria $E_{00}, E_{01}, E_{10}, E_{11}$, and $E_{22 a}$ do not depend on $d$ and are as described in the previous section. As can be seen in Table 1 the equilibria $E_{12}$ and $E_{21}$ for $d=0$ correspond to the case where one patch is empty and the other is at the two-species equilibrium. Finally, for $d=0$ the equilibria $E_{22 b}$ and $E_{22 c}$ represent the cases where one patch has only prey while the other has a twospecies equilibrium. (Note that for consistent notation for $d=0$ the equilibria $E_{12}$ and $E_{21}$ could be denoted $E_{02}$, $E_{20}$, and $E_{22 b}$ and $E_{22 c}$ as $E_{12}$ and $E_{21}$, but this can potentially lead to confusion. I will not do so and use the notation as given in Table 1 also for $d=0$.) Since all equilibria of (1) are unstable for the choice of parameters used, all equilibria of (2) are unstable for small $d$. For small, positive $d$ the equilibria $E_{22 b}$ and $E_{22 c}$ have a negative predator density in one of the patches and no nontrivial equilibria exist outside $D$.

Six other $\omega$-limit sets exist for $d=0$ which consist of combinations of a limit cycle in one patch and an equilibrium in the other. Because (1) has three equilibria, three different combinations can be formed of a limit cycle and an equilibrium and because every combination has an $R$ image there are six such $\omega$-limit sets in total. For the choice of parameters used all equilibria of (1) are unstable; hence, for small $d$ no stable $\omega$-limit sets formed out of a combination of equilibrium and limit cycle exist. Four of these limit cycles could be detected with the continuation procedure used and all four are unstable for small $d$. Two $\omega$-limit sets at $d=0$, corresponding to the combination of the limit cycle and the one species equilibrium and its $R$ image, could not be continued but simulations indicated that these sets do not give rise to attractors.

Finally, the dynamics can exhibit limit cycles in both patches. For $d=0$ the oscillations in the patches can have all possible phase differences; hence, a family of different limit cycles exists, all with the same period but a different phase difference. Together these limit cycles form a torus. One of the limit cycles on the torus is the F-cycle $\Gamma$. The F-cycle and a limit cycle on which the fluctuations in the patches are in counterphase, i.e., have a phase difference of $\pi$, are the only limit cycles stemming from the torus that could be continued for small $d$. This second limit cycle does not lie in $D$ but the $R$ image of the orbit of this limit cycle is the orbit itself and therefore the orbit is symmetrical under $R$. Such a symmetrical cycle is called an S-cycle in the terminology of $\mathbb{Z}_{2}$-equivariant systems (Kuznetsov, 1995). The F-cycle $\Gamma$ is stable, while the S-cycle is unstable for small values of $d$. This makes $\Gamma$ the only attractor for very small migration rates.

It is often assumed that two weakly coupled oscillating populations behave as two decoupled oscillators (Taylor, 1990; Harrison and Taylor, 1997, Stacey et al., 1997). If this were true in our model some of the $\omega$-limit sets that exist for zero migration rates, other than the limit cycle $\Gamma$ on which the oscillations are in perfect synchrony, should be stable for small migration rates. For system (2) this does not hold: none of the $\omega$-limit sets that exist for $d=0$ is stable for small $d$, other than the limit cycle $\Gamma$. Weak coupling always caused the dynamics of the two patches always to become synchronized.

\subsection{One Patch without Prey}

Figure 1 gives the bifurcation structure within the face $N_{2}=0$, i.e., when no prey is present in patch 2 . If the patches are not coupled, three $\omega$-limit sets with nonnegative prey densities exist within this face, these being the prey-only equilibrium $E_{10}$, the predator-prey equilibrium $E_{21}$, and a limit cycle formed out of the combination of a limit cycle in patch 1 while patch 2 is empty. For increasing $d$ this limit cycle shrinks and connects to equilibrium $E_{21}$ in a supercritical Hopf bifurcation (labeled H1 in Fig. 1).

The equilibrium $E_{21}$ is unstable as it has an eigenvector transversal to the face $N_{2}=0$ associated with a positive eigenvalue: at this equilibrium a small number of prey introduced in patch 2 will increase in numbers. All $\omega$-limit sets apart from the F-cycle $\Gamma$ are unstable for small $d$. For larger $d, E_{21}$ goes through a second Hopf bifurcation (H2). The limit cycle that branches off attracts within the face $N_{2}=0$ but is unstable with respect to the introduction of prey in patch 2 . When $d$ is increased further, first a transcritical bifurcation of equilibria (TE) could be detected on $E_{21}$ (the equilibrium $E_{22 b}$ leaves the positive state space here; this will be discussed in more detail the next section). The equilibrium is still unstable within the face $N_{2}=0$.

For larger $d$ the limit cycle that came into existence at (H2) goes through a transcritical bifurcation of cycles (TC) in which it gains stability: the limit cycle is now stable against introduction of small amounts of prey $N_{2}$. The biological interpretation for this is that for large 


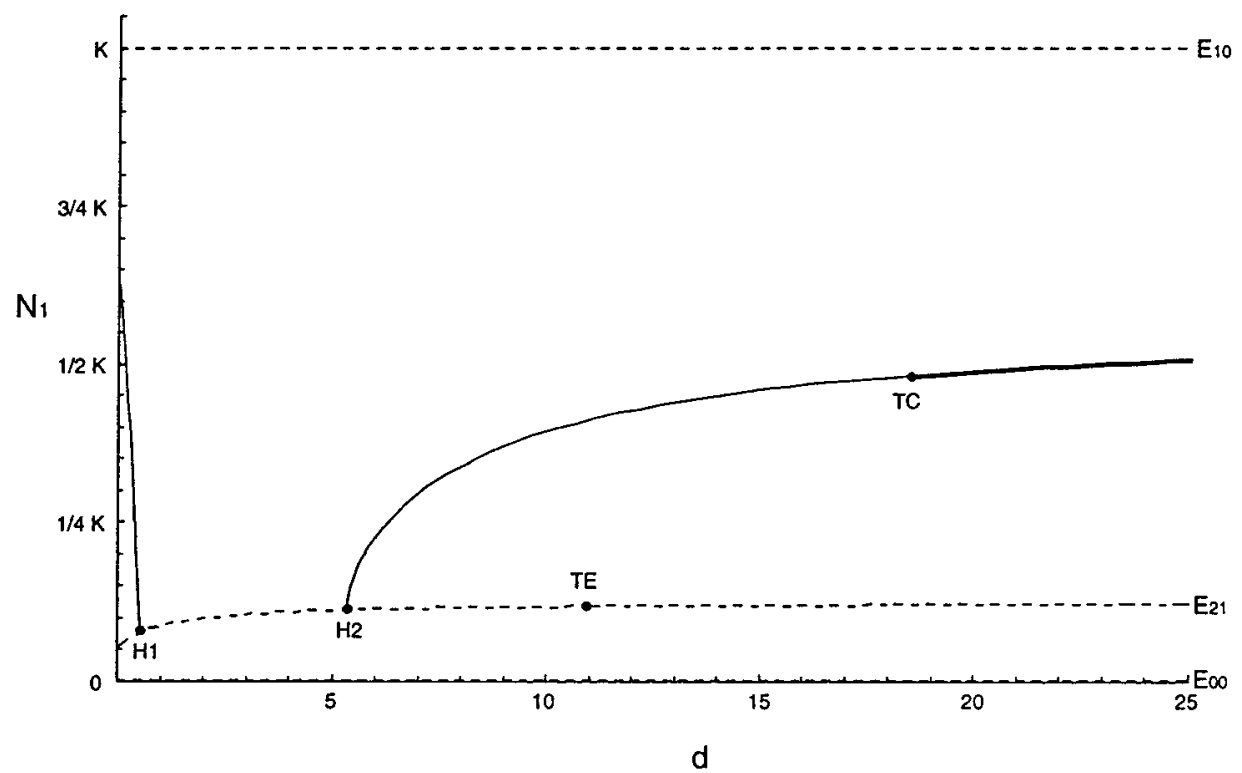

FIG. 1. A one-parameter bifurcation diagram in $d$ for (2) in which only the branches in the face $N_{2}=0$ are drawn. On the vertical axis the value of $N_{1}$ in the equilibria and the maximum of $N_{1}$ for limit cycles are given. Dashed lines represent unstable equilibria, thin drawn lines unstable limit cycles, and thick drawn lines stable limit cycles. Black dots indicate bifurcations. Labels: H1, H2: Hopf bifurcations, TE: transcritical bifurcation of equilibria, TC: transcritical bifurcation of cycles. Parameter values: $b=9.96, K=2 b, \mu=r=1$.

predator migration rates, prey which is introduced in patch 2 will be consumed quickly by the predators which have migrated into patch 2 from patch 1 . Therefore, no prey population can persist in this patch. No other $\omega$-limit sets with nonnegative prey densities were found.

\subsection{Small Predator Migration Rates}

Figure 2 summarizes the results on the bifurcation structure in $\mathbb{R}_{+}^{4}$ for small predator migration rates. Some of the $\omega$-limit sets described in the previous subsection exist for small predator migration rates and are also

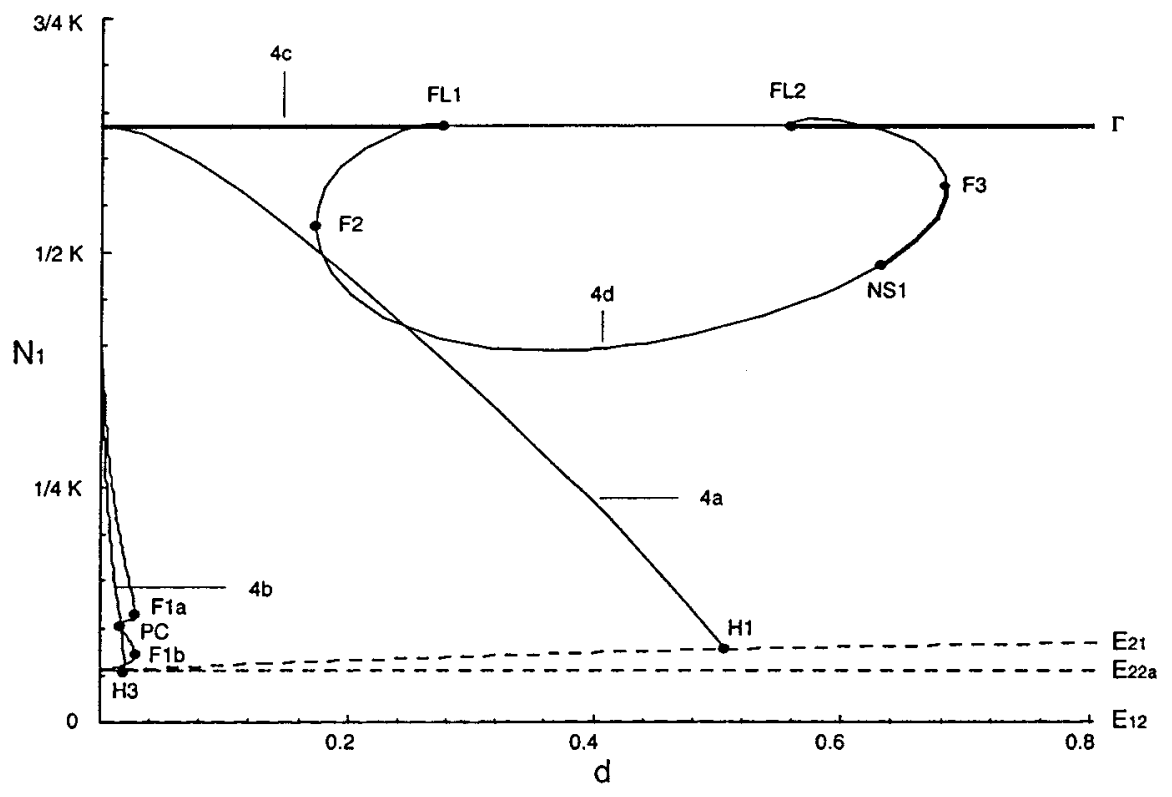

FIG. 2. A one-parameter bifurcation diagram for (2) for small values of $d$, giving the value of $N_{1}$ in the equilibria and the maximum of $N_{1}$ for limit cycles. The legends for lines and labels is given in Fig. 1; lines and labels refer to the phase portraits in Fig. 3. Additional labels: F1a F1b, F2, F3: fold bifurcations of limit cycles, FL1, FL2: flip bifurcations, H3: Hopf bifurcation, NS1: Neimark-Sacker bifurcation, PC: pitchfork bifurcation of limit cycles. Parameter values are as in Fig. 1. 


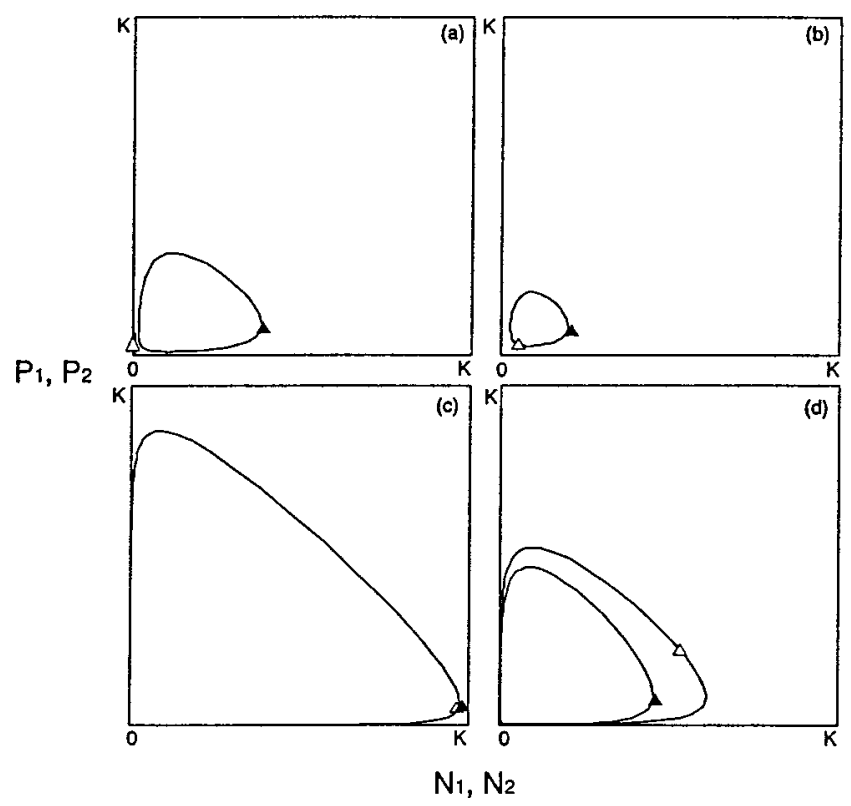

FIG. 3. Phase portraits for a number of limit cycles in Fig. 2. The black and open dots given the position of the densities at the same moment for the different patches. For the symmetrical limit cycle in (c) the solid and open triangles superimpose. Parameter values are as in Fig. 1, except $d$ which takes the value 0.4 (a), 0.0136 (b), and 0.4 (d). The phase portrait in (c) can be obtained for all values of $d$.

depicted in this figure. For instance, the limit cycle that connects to $E_{21}$ in Hopf bifurcation (H1) is shown. Figure $3 a$ gives the phase portrait of this limit cycle.

For very small values of $d$ the predator densities in the equilibria $E_{22 b}$ and $E_{22 c}$ are negative in one of the patches and therefore not shown in the figure. The equilibrium $E_{22 a}$ is always positive. Because the model parameters are chosen such that the limit cycle $\Gamma$ exists, the equilibrium $E_{22 a}$ is unstable for all $d$. For very small $d$ a S-cycle connects to $E_{22 a}$ in Hopf bifurcation (H3). As the phase portrait in Fig. $3 \mathrm{~b}$ indicates, the local oscillations are in counterphase and have a phase difference of $\pi$. Due to the symmetry this limit cycle has to approach the equilibrium from a direction perpendicular to $D$ (Kuznetsov, 1995).

The only stable $\omega$-limit set that exists for small $d$ is the F-cycle $\Gamma$ (see Fig. 3c for phase portrait). This limit cycle is stable for small $d$ and then loses stability with increasing $d$ in a flip bifurcation (FL1). This is an important bifurcation in terms of biology because the synchronous F-cycle $\Gamma$, which for small $d$ is an attractor, becomes unstable and $\omega$-limit sets on which the dynamics are asynchronous are connected to the synchronous limit cycle $\Gamma$ at this bifurcation. For still larger $d$ the reverse happens when $\Gamma$ regains stability, again in a flip bifurcation (FL2). In the domain where the synchronous limit cycle $\Gamma$ is unstable, no synchronous long-term dynamics exist and in this region other attractors must exist on which the dynamics are asynchronous.

To find the asynchronous attractors I continued the $\omega$-limit sets that connect to $\Gamma$ in the flip bifurcations FL1 and FL2. both flip bifurcations are subcritical: close to the bifurcation an unstable S-cycle with a period of approximately twice the period of $\Gamma$ exists while $\Gamma$ is stable. The unstable S-cycles formed in the flip bifurcations disappear in fold bifurcations F2 and F3. In the fold bifurcation F3 the unstable S-cycle meets a stable S-cycle with a period of roughly twice the period of $\Gamma$; its phase portrait is given in Fig. 3d. This S-cycle is stable only in the vicinity of bifurcation point F3 and loses stability for decreasing $d$ in a Neimark-Sacker bifurcation (NS1). In this bifurcation an invariant torus forms around the limit cycle and the dynamics become quasi-periodic.

Figure 2 does not show an attractor in the $d$-region where $\Gamma$ is unstable. The dynamical behavior in this region can be clarified using the two-parameter bifurcation diagram shown in Fig. 4, in which the fold bifurcations of cycles (F2, F3), the flip bifurcations (FL1, FL2), and the Neimark-Sacker bifurcations (NS1) are continued in the two-parameter space $(d, K)$. It can be seen that for decreasing $K$ ( $K$ equals $2 b$ in Fig. 2 ) the two flip bifurcation points meet and disappear. For $K$ chosen lower than the minimum of the flip curve in Fig. 4 the arc connected to $\Gamma$ at FL1 and FL2 in Fig. 2 closes and forms an isolated closed curve. The Neimark-Sacker bifurcation and the fold bifurcation meet in one point. In this codimension 2 bifurcation point, known as a strong resonance $1: 1$ point, the two asymmetrical limit cycles each have two multipliers of value 1 at the point of collision. It is known that two curves, each corresponding to a homoclinic tangency, are attached to this point (Arrowsmith and Place, 1990; Kuznetsov, 1995). These curves (not shown) lie above the Neimark-Sacker curve in Fig. 4. Although the exact unfolding around this bifurcation is not known, the following scenario is possible (Kuznetsov, 1995): in the parameter region between the homoclinic tangency curves the stable and unstable manifolds of the saddle intersect. In this homoclinic tangle the intersections of the stable and unstable manifolds of the saddle cycle intersect and form a horseshoe map. The dynamics of a horseshoe map can be chaotic (Wiggins, 1990). The dynamics is such that the system stays near the saddle for a number of iterations followed by an excursion away from the saddle, then to return again to the neighborhood of the saddle, giving rise to intermittent chaos (Bergé et al., 1984). The dynamics are chaotic in the sense that the sequence of the numbers of iterations spent in the neighborhood of the saddle can take any form. 


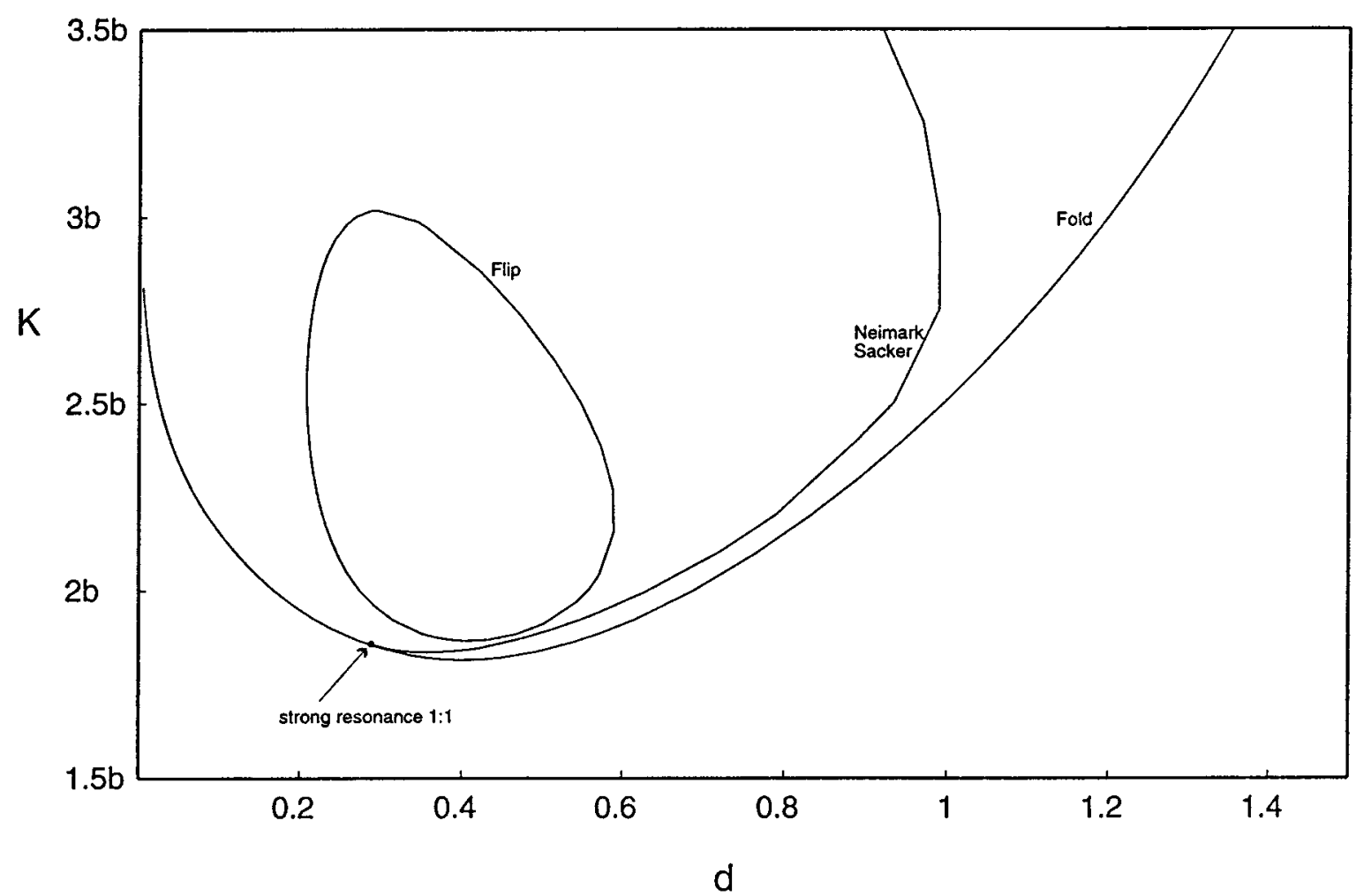

FIG. 4. A two-parameter bifurcation diagram for (2) in $d$ and $K$ giving the curves for the flip, Neimark-Sacker, and the fold bifurcations of some of the bifurcations in Fig. 2. See text for explanation. At the point where the Neimark-Sacker curve meets the curve for the tangent bifurcation a strong resonance 1:1 point can be found. Parameters (except $K$ ) are as in Fig. 1.

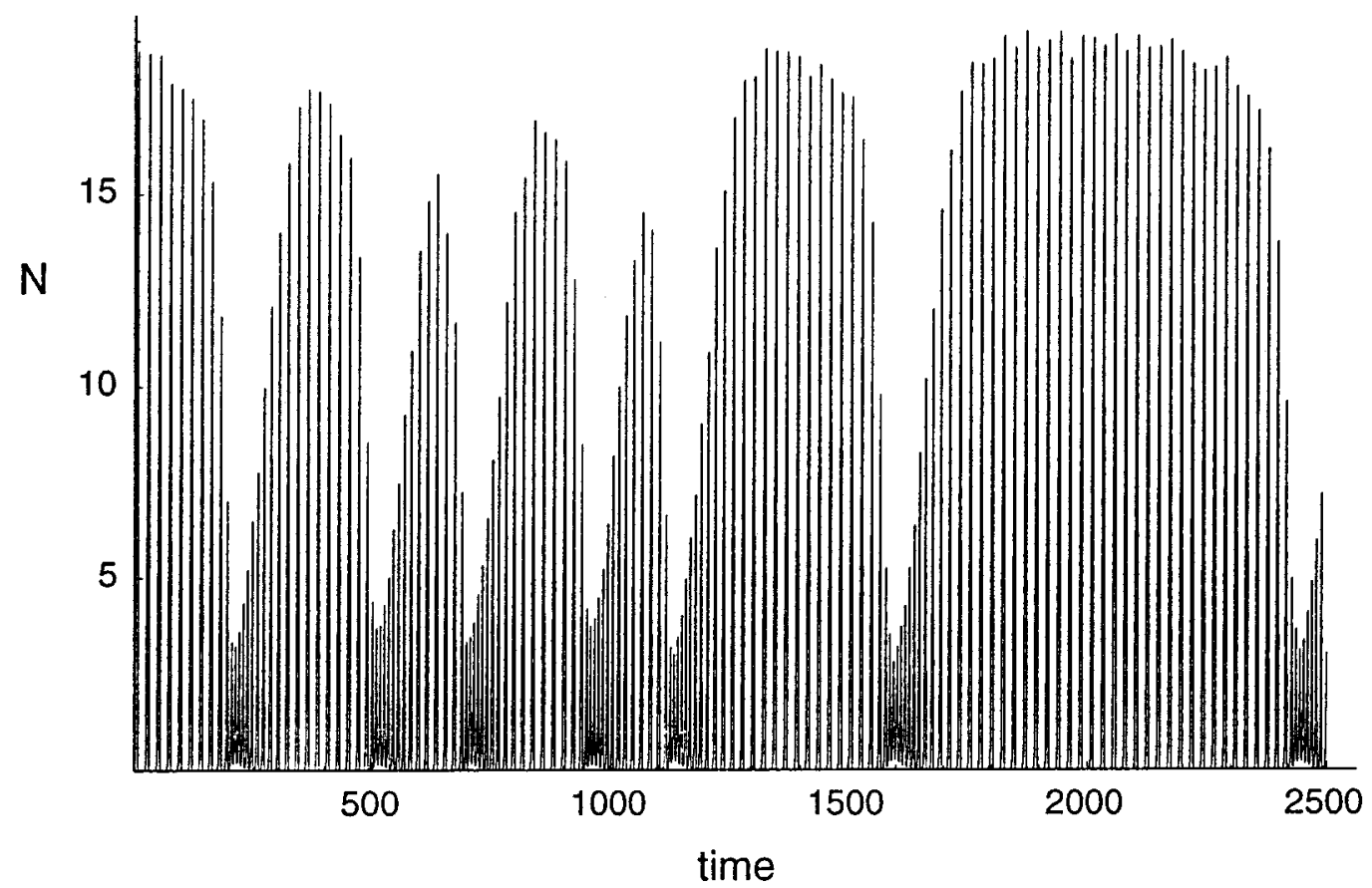

FIG. 5. The average prey density, $N=\left(N_{1}+N_{2}\right) / 2$, over time for a chaotic solution of system (2). The orbit visits the synchronous orbit for intervals of irregular length. Parameter values: $b=9.96, K=25, \mu=r=1, d=0.5$. 
For parameter values close to the homoclinic tangency curves, intermittently chaotic dynamics are indeed observed with irregular visits to the saddle cycle, mixed with excursions on which the amplitude of the oscillations are decreased (Fig. 5). This suggests that the homoclinic tangle formed by the overlapping stable and unstable manifolds exists and persists under small changes of parameters as will the chaotic dynamics. For a further decrease of $d$ the homoclinic tangle disappears. Before it disappears totally, $\Gamma$ regains stability in a subcritical flip bifurcation (FL1). Finally, the two unstable S-cycles grow toward each other, collide, and disappear in fold bifurcation F2.

The carrying capacity $K$ asserts a stabilizing influence on the dynamics. By increasing $K$ the amplitude of the oscillations increases while the minimum densities over the limit cycle decrease. For increasing $K$ the curve for the flip bifurcation closes: for large carrying capacities the limit cycle $\Gamma$ is stable. The curve for the fold bifurcation widens and encloses a region where asynchronous attractors exist. Simulations showed that asynchronous attractors of the types as described in this subsection do exist in a large part of this region.

\subsection{Large Predator Migration Rates}

Figure 6 shows some of the stable and unstable $\omega$-limit sets for large values of $d$. Note that throughout this range the F-cycle $\Gamma$ is stable but that this limit cycle does not connect to the limit cycle in the face $N_{2}=0$. The equilibrium $E_{22 a}$ is unstable for all values of $d$. From this equilibrium the unstable equilibria $E_{22 b}$ and $E_{22 c}$ branch off in a pitchfork bifurcation (PE). With increasing $d$ the equilibria move through the positive state space toward the faces and leave the positive state space through, respectively, $E_{21}$ and $E_{12}$ in transcritical bifurcations of equilibria, after which $E_{22 b}$ and $E_{22 c}$ are negative and unstable. For increasing $d$ a limit cycle enters the positive state space through the limit cycle that branched off $E_{21}$ in a transcritical bifurcation of cycles. In this bifurcation the limit cycle transfers its stability to the limit cycle that lies in the face $N_{2}=0$. The stable manifold of the now unstable limit cycle that entered the positive state space forms the separatrix between the domains of attraction of the stable limit cycle in the face $N_{2}=0$, the stable limit cycle in the face $N_{1}=0$, and $\Gamma$. This limit cycle was formed in the negative state space in Hopf bifurcation $\mathrm{H} 4$ (not shown).

When the transcritical bifurcations of equilibria and the Hopf bifurcation on $E_{21}(\mathrm{H} 4)$ are continued in a two-parameter space, the curves corresponding to these bifurcations cross (Fig. 7, see Klebanoff and Hastings (1994) and Kuznetsov and Rinaldi (1996) for a detailed description of the dynamics around this codimension 2 bifurcation). At the codimension 2 point an equilibrium exists with two purely imaginary eigenvalues and a real eigenvalue that equals zero. The eigenvectors associated

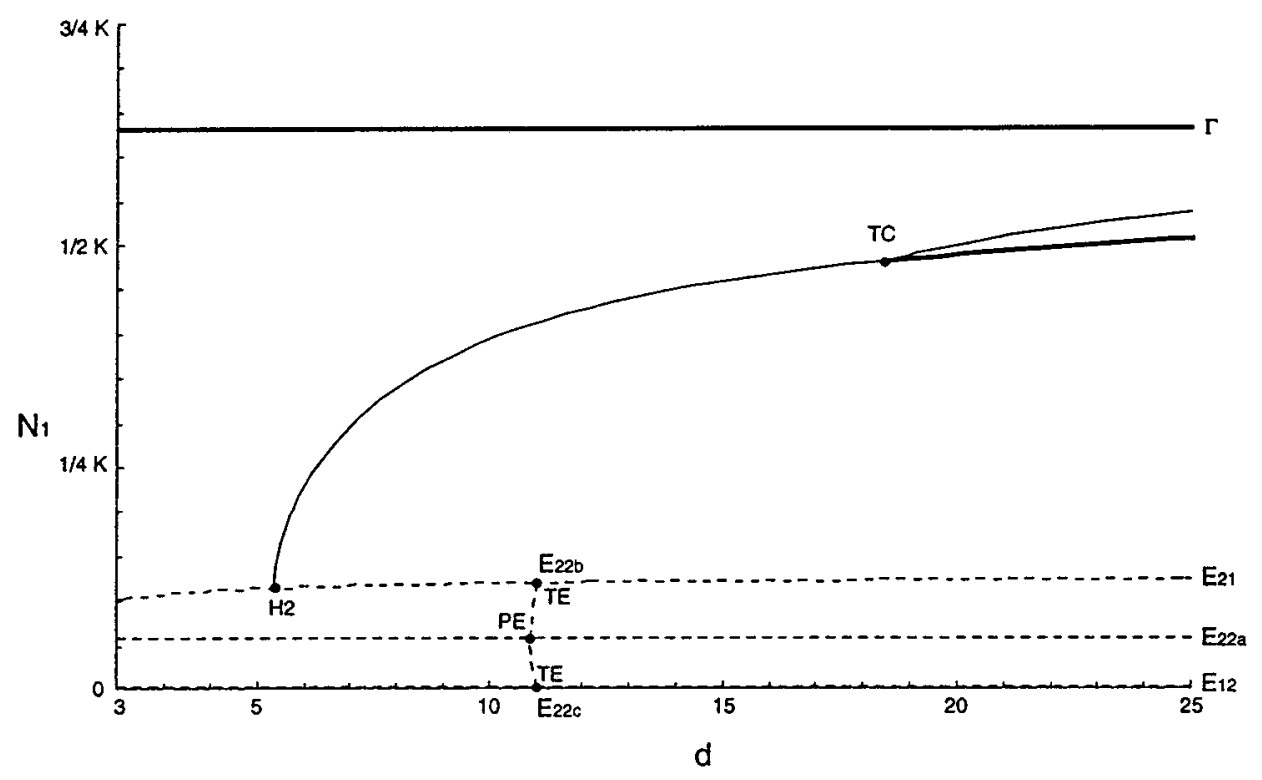

FIG. 6. A one-parameter bifurcation diagram for (2) for large values of $d$, giving the value of $N_{1}$ in the equilibria and the maximum of $N_{1}$ for limit cycles. Dashed lines represent unstable positive equilibria, thin drawn lines unstable limit cycles, and thick drawn lines stable positive limit cycles. Black dots indicate bifurcations. Labels: H2: Hopf bifurcation, PE: pitchfork bifurcation of equilibria, TC: transcritical bifurcation of limit cycles, TE: transcritical bifurcation of equilibria. Parameter values are as in Fig. 1. 
with the imaginary eigenvalues lie in the invariant face and the eigenvector associated with the eigenvalue at zero is transversal to this face. At this codimension 2 point simultaneously a Hopf bifurcation takes place in the invariant face with a transcritical bifurcation in which an equilibrium crosses the invariant face. The curves corresponding to a Hopf bifurcation at the "entering" equilibrium and a transcritical bifurcation of cycles are attached to this codimension 2 bifurcation point (Klebanoff and Hastings, 1994; McCann and Yodzis, 1995; Jansen, 1995b; Kuznetsov and Rinaldi, 1996). Figure 7 shows these curves. Also, the curve for the Neimark-Sacker bifurcation is attached to this pont (Klebanoff and Hastings, 1994) (not shown in Fig. 7). Note that the curve for the pitchfork bifurcation does not go through the codimension 2 point since it is associated with equilibrium $E_{22 a}$, which cannot move out of $\mathbb{R}_{+}^{4}$ through $E_{12}$ or $E_{21}$.
Figure 7 shows that for values of $K$ lower than the codimension 2 bifurcation point a stable attractor in the face $N_{2}=0$ can be formed through a different scenario (Van der Laan and Hogeweg, 1995). When a cross section through Fig. 7 is being made for fixed $K$ below the codimension 2 bifurcation point, the following sequence of bifurcations takes place for increasing $d$ : first a pitchfork bifurcation of the unstable equilibrium $E_{22 a}$ $(\mathrm{PE})$ is encountered in which the unstable equilibria $E_{22 b}$ and $E_{22 c}$ are formed, as shown in Fig. 6. These two equilibria gain stability in Hopf bifurcation (H4) where unstable limit cycles branch off. The stable manifolds of these limit cycles form the separatrices between the domains of attraction of $E_{22 b}, E_{22 c}$, and the attracting F-cycle $\Gamma$. For larger $d$ the stable equilibria can leave the positive state space through, respectively, $E_{12}$ and $E_{21}$ in transcritical bifurcations. The stability then is transferred from $E_{22 b}$ and $E_{22 c}$ to $E_{12}$ to $E_{21}$. For $d$ still larger, $E_{12}$

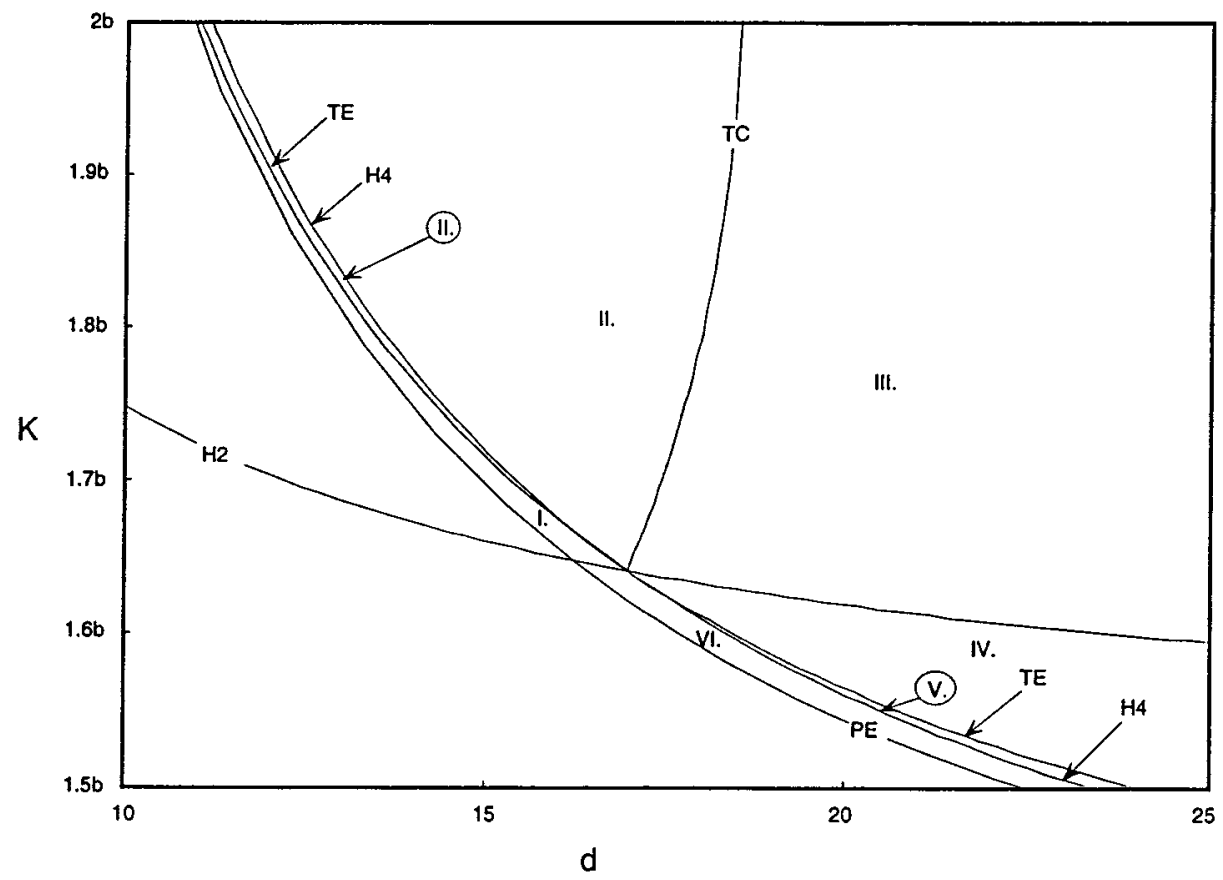

FIG. 7. A two-parameter bifurcation diagram for (2) in $d$ and $K$ giving the curves for the Hopf bifurcations on equilibria $E_{21}$ and $E_{12}$ (H2), the Hopf bifurcations on equilibria $E_{22 b}$ and $E_{22 c}$, (H4), the transcritical bifurcations of the equilibria $E_{22 b}$ with $E_{21}$ and $E_{22 c}$ with $E_{12}$ (TE), the transcritical bifurcation of limit cycles (TC), and the pitchfork bifurcation on equilibrium $E_{22 a}(\mathrm{PE})$. At the point where the curves $\mathrm{H} 2$, H4, TE, and TC cross (note that TE and $\mathrm{H} 4$ actually cross), a codimension 2 bifurcation point exists. Around the codimension 2 bifurcation, seven different regions exist with different arrangements of limit cycles and equilibria. In region I, two unstable limit cycles and two unstable equilibria lie in the face $N_{i}=0$; the equilibria $E_{22 b}$ and $E_{22 c}$ are positive and unstable. In region II, two unstable limit cycles and two unstable equilibria lie in the faces; equilibria $E_{22 b}$ and $E_{22 c}$ are nonpositive. In region III, two unstable limit cycles and two unstable equilibria lie in the faces; two nonpositive limit cycles branched off $E_{22 b}$ and $E_{22 c}$; in part of the region these limit cycles are stable. In region IV, stable limit cycles and unstable equilibria lie in the faces; two unstable limit cycles exist in positive state space. In region $\mathrm{V}$, two stable equilibria lie in the faces; two unstable limit cycles exist in positive state space. In region VI, two unstable equilibria lie in the faces; two stable equilibria and two unstable limit cycles lie in positive state space. In region VII, two unstable equilibria lie in the faces and two unstable equilibria lie in positive state space. In all regions the synchronous limit cycle is stable and equilibrium $E_{22 a}$ is unstable. Parameters (except $K$ ) are as in Fig. 1. 
and $E_{21}$ can go through Hopf bifurcations (H2) in which stable limit cycles in the faces $N_{i}=0$ come into existence.

This scenario is somewhat surprising and relates to the classic result by Segel and Jackson (1972) that in spatial predator-prey systems equilibria cannot lose their stability other than in a Hopf bifurcation. This seems to imply that no spatial pattern can be formed from equilibria but this is not the case: once the equilibrium $E_{22 a}$ has become unstable in a Hopf bifurcation this equilibria can go through a pitchfork bifurcation in which two new equilibria are formed, which then can regain stability. In this way the "crystal lattice" type spatial patterns can form in simulations on large grids (Hassell et al., 1991; Van der Laan and Hogeweg, 1995; De Roos et al., 1998).

\section{DISCUSSION}

The two-patch model presented in this paper is a very simple spatial model for oscillating populations. Despite its simplicity the model has a rich dynamical behavior and the model portrays many characteristics of spatial populations. For instance, the model demonstrates that both synchronous and asynchronous dynamics arise naturally. Synchronous oscillations can be found for very large and very small predator migration rates. Stable asynchronous oscillations can be found for a relatively small domain of intermediate predator migration rates. As presumed in metapopulation theory, asynchronous dynamics do arise naturally in systems of coupled oscillating populations even if the environments in both patches are the same. However, asynchronous dynamics do not arise from very loosely coupled oscillators but are brought about by the regulatory forces that act on the local populations and the spatial interaction. If the coupling is too strong or too weak, the oscillations phase lock and synchronous oscillations result. The phase locking is a not just a consequence of the initial conditions, as is sometimes assumed (Ranta et al., 1999), but is a stable state of the system and seems the default state for weak coupling. Very loosely coupled oscillating populations always showed phase-locked population dynamics. This finding seems to disagree with the opinion expressed in the metapopulation literature (e.g., Taylor, 1990; Harrison and Taylor, 1997, Stacey et al., 1997). It would be interesting to know whether this is generally true for weakly diffusively coupled identical oscillators and how such coupled oscillators behave under added environmental noise.

When more than two patches are coupled, the asynchronous dynamics generally occur for a larger range of migration rates (Jansen and De Roos, 2000; Jansen and Lloyd, 2000). The main reason for this is that, in larger systems, the coupling between patches decreases with the distance between the patches. For large migration rates, which in a two-patch system would only result in phaselocked dynamics, it is possible to find patches which have just the right amount of coupling to give rise to the symmetry breaking that leads to asynchronous dynamics. This argument can be made mathematically precise by considering the stability of solutions for which there are no differences between the patches (i.e., the synchronous solutions which live in the subspace $D$ ). In many cases the existence of asynchronous solutions can be deduced from their presence in a two-patch model. Once results on stability have been obtained for a two-patch model, a generalization to systems with more patches is straightforward for all possible geometrical arrangements of the patches (Jansen and De Roos, 2000; Jansen and Lloyd, 2000). Synchronous solutions form a natural starting point for the investigation of the dynamics of spatial systems. This also provides a direct link to much experimental work, which are often continuous-time, multipatch systems (Janssen et al., 1997; Gonzalez et al., 1998; Holyoak and Lawler, 1996a b; Holyoak, 2000). This makes two-patch models a versatile tool for the investigation of the dynamics of spatial systems.

The same population dynamics that can drive wellmixed populations to extinction can lead to population persistence if the movement of individuals is limited (De Roos et al., 1991; Hassell et al., 1991). Also in this model asynchronous dynamics oscillate with a smaller amplitude than the well-mixed system would. This can happen in two possible ways: if the predator migration rates are large, the prey in one patch can become extinct because of the flow of predators from another patch. This is the equivalent of the crystal lattice pattern observed in coupled map lattice models of host parasitoid dynamics (Hassell et al., 1991; Comins et al., 1992; Van der Laan and Hogeweg, 1995; De Roos et al., 1998). The other possibility for intermediate predator migration rates is that the periodic, quasi-periodic, or chaotic dynamics arise over which the amplitudes of the oscillations are reduced. This could well be similar to the spiral chaos patterns in the coupled map lattice models (Hassell et al., 1991; Comins et al., 1992). The asynchrony of local dynamics has also been demonstrated in persisting experimental predator-prey systems (Van de Klashorst et al., 1992; Lingeman and Van de Klashorst 1992; Holyoak, in press; Holyoak and Lawler, 1996a,b; Janssen et al., 1997). Interestingly, in the experimental system studied by Van de Klashorst et al. (1992) the densities in the different compartments of the experimental system fluctuated in phase for about a year, and then switched to a different type of dynamics in which the 
densities were out of phase for another year. This suggests that spatial oscillating systems have stable synchronous and asynchronous modes, which exist simultaneously. This feature has been demonstrated here and in other models (Hassell et al., 1991; Adler, 1993; Jansen, 1995a).

In well-mixed predator-prey systems an increase in the prey's carrying capacity leads to oscillations with increased amplitudes, bringing populations ever close to the boundaries of extinction (Rosenzweig, 1971, 1972). As shown here, in spatial systems the amplitudes in the oscillations can be less extreme through lasting differences between the local densities, preventing the populations to reach densities where extinction is likely to occur. The mechanism that maintains differences works as follows: if two patches start out with the same predator density but a difference in the minimum prey density, the patch with less prey will reach a higher maximum prey density and takes longer to complete a cycle. After prey density has increased and most prey are consumed, the predator density will lag behind that in the other patch and this patch will a this point in time have more predators than the other. During the near exponential decay in predator densities, this patch will export predators to the other patch. As a result, the prey density in the other patch decreases faster. At the beginning of the next cycle the patch that has less prey to begin with now has more prey and vice versa. For intermediate predator migration rates the magnitude of the differences increases from cycle to cycle and this destabilizes the synchronous oscillation. For very large predator migration rates the differences are mixed out; for very small migration rates not enough predators are exported to destabilize the synchronous oscillation. For this mechanism to operate the phase of near-exponential prey growth needs to be sufficiently large and the prey migration low or absent in order for difference in prey densities to build up.

Thus, the large fluctuations that result from enrichment also offer a mechanism for the reduction of the fluctuations in an ensemble of patches (see also Jansen, 1995a). An increase in the carrying capacity in a spatial predator-prey system for small carrying capacities gives rise to an increase in the period of near-exponential prey growth. This allows for differences in densities and asynchronous dynamics to be maintained and will result in oscillations with a reduced amplitude compared to synchronous dynamics. A further increase in carrying capacities causes the amplitude of the asynchronous dynamics to saturate and extreme enrichment has little effect on the population densities once asynchronous dynamics have developed (Jansen, 1995a). In predatorprey systems in which the prey is not self limited i.e., can show exponential growth, the well-mixed system will eventually drive itself to extinction (Jansen 1994, 1995a, Weisser et al., 1997). Also in this case, limited movement of predators and prey can regulate the populations and prevent the extinction of populations.

The parameter region for which spatial dynamics can prevent extinction is relatively small in the two-patch model analyzed here. In systems with more patches this region expands (Jansen and Lloyd, 2000). Therefore, the large fluctuations that seem to abound in small predator-prey systems will be much reduced in lager spatial systems (Jansen and De Roos, 2000). Spatial interactions can resolve Rosenzweig's paradox of enrichment.

\section{ACKNOWLEDGMENTS}

I am indebted to Pavel Brunovski for many inspiring discussions and to Odo Diekmann and Alan Hastings for comments on the manuscript. Support of the Austrian Forschungs Förderungs Fonds No. 8043 is gratefully acknowledged. Mous Sabelis and Robert Payne were instrumental in the eventual publication of this work.

\section{REFERENCES}

Adler, F. 1993. Migration alone can produce persistence of hostparasitoid models, Am. Nat. 141, 642-650.

Allen, J. C., Schaffer, W. M., and Rosko, D. 1993. Chaos reduces species extinction by amplifying local population noise, Nature 364, 229-232.

Arrowsmith, D. K., and Place, C. M. 1990. "An Introduction to Dynamical Systems," Cambridge Univ. Press, Cambridge.

Ashwin, P., and Swift, J. W. 1992. The dynamics of $n$ weakly coupled identical oscillators, J. Nonlinear Sci. 2, 69-108.

Bergé, P., Pomeau, Y., and Vidal, C. 1984. "Order within Chaos," Wiley, Chicester.

Comins, H. N., Hassell, M. P., and May, R. M. 1992. The spatial dynamics of host-parasitoid systems, J. Anim. Ecol. 61, 735-748.

Crowley, P. H. 1978. Effective size and persistence of ecosystems, Oecologia 35, 185-195.

Czaran, T. 1998. "Spatiotemporal Models of Population and Community Dynamics," Chapman and Hall, London.

De Roos, A. M., McCauley, E., and Wilson, W. G. 1991. Mobility versus density limited predator-prey dynamics on different spatial scales, Proc. R. Soc. London B 246, 117-122.

De Roos, A. M., McCauley, E., and Wilson, W. G. 1998. Pattern formation and the spatial scale of interaction between predators and their prey, Theor. Popul. Biol. 53, 108-130.

Gause, G. F. 1934. "The Struggle for Existence," William and Wilkins, reprinted in 1964 by Hafner, New York.

Gilpin, M., and Hanski, I. 1991. Metapopulation dynamics: Empirical and theoretical investigations, Biol. J. Linn. Soc. 42.

Gonzalez, A., Lawton, J. H., Gilbert, F. S., Blackburn, T. M., and Evans-Freke, I. 1998. Metapopulation dynamics, abundance, and distribution in a microecosystem, Science 281, 2045-2047. 
Grenfell, B. T., Wilson, K., Finkenstadt, B. F., Coulson, T. N., Murray, S., Albon, S. D., Pemberton, J. M., Clutton-Brock, T. H., and Crawley, M. J. 1998. Noise and determinism in synchronized sheep dynamics, Nature 394, 674-677.

Gyllenberg, M., Söderbacka, G., and Ericsson, S. 1993. Does migration stabilize local population dynamics? Analysis of a discrete metapopulation model, Math. Biosci. 118, 25-49.

Hanski, I. 1991. Single-species metapopulation dynamics: Concepts, models and observations, Biol. J. Linn. Soc. 42, 17-38.

Hanski, I., and Gilpin, M. 1997. "Metapopulation Biology: Ecology, Genetics and Evolution," Academic Press, San Diego.

Harrison, S., and Taylor, A. D. 1997. Empirical evidence for metapopulation dynamics, in "Metapopulation Biology: Ecology, Genetics and Evolution" (I. Hanski and M. Gilpin, Eds.), Academic Press, San Diego.

Hastings, A. 1993. Complex interactions between dispersal and dynamics-lessons from the coupled logistics equations, Ecology $\mathbf{7 4}$, 1362-1372.

Hastings, A., and Harrison, S. 1994. Metapopulation dynamics and genetics, Ann. Rev. Syst. Ecol. 25, 167-188.

Hassell, M. P., Comins, H. N., and May, R. M. 1991. Spatial structure and chaos in insect population dynamics, Nature 363, 252-258.

Hofbauer, J., and Sigmund, K. 1998. "Evolutionary Games and Population Dynamics," Cambridge Univ. Press, Cambridge.

Holyoak, M. 2000. The effects of nutrient enrichment on predator-prey metapopulation dynamics, J. Anim. Ecol. 69, 985-997.

Holyoak, M., and Lawler, S. P. 1996a. Persistence of an extinctionprone predator-prey interaction through metapopulation dynamics, Ecology 77, 1867-1879.

Holyoak, M., and Lawler, S. P. 1996b. The role of dispersal in predator-prey metapopulation dynamics, J. Anim. Ecol. 65, 640-652.

Hudson, P. J., and Cattadori, I. M. 1999. The Moran effect: A cause of population synchrony, Trends. Ecol. Evol. 14, 1-2.

Huffaker, C. B. 1958. Experimental studies on predation: Dispersion factors and predator-prey oscillations, Hilgardia 27, 343-383.

Jansen, V. A. A. 1994. "Theoretical Aspects of Metapopulation Dynamics," Ph.D. thesis, Leiden University, The Netherlands.

Jansen, V. A. A. 1995a. Regulation of predator-prey systems through spatial interactions: A possible solution to the paradox of enrichment, Oikos 74, 384-390.

Jansen, V. A. A. 1995b. Effects of dispersal in a tri-trophic metapopulation model, J. Math. Biol. 34, 195-224.

Jansen, V. A. A. 1999. Phase locking: Another cause of synchronicity in predator-prey systems, Trends Ecol. Evol. 14, 278-279.

Jansen, V. A. A., and Lloyd, A. L. 2000. Local stability analysis of spatially homogeneous solutions of multi-patch systems, J. Math. Biol. 41, 232-252.

Jansen, V. A. A., and De Roos, A. M. 2000. The role of space in reducing population cycles, in "The Geometry of Ecological Interactions: Simplifying Spatial Complexity" (U. Dieckmann, R. Law, and J. A. J. Metz, Eds.), Cambridge Univ. Press, Cambridge.

Janssen, A., Van Gool, E., Lingeman, R., Jacas, J., and Van de Klashorst, G. 1997. Metapopulation dynamics of a persisting predator-prey system in the laboratory: Time series analysis, Exp. Appl. Acarol. 21, 415-430.

Khibnik, A. I., Kuznetsov, Y. A., Levitin, V. V., and Nikolaev, E. V. 1993. Continuation techniques and interactive software for bifurcation analysis of ODEs and iterated maps, Physica D 62, 360371.

Klebanoff, A., and Hastings, A. 1994. Chaos in three species food chains, J. Math. Biol. 32, 427-451.
Koenig, W. D. 1999. Spatial autocorrelation of ecological phenomena, Trends Ecol. Evol. 14, 22-26.

Kuznetsov, Y. A. 1995. "Elements of Applied Bifurcation Theory," Springer-Verlag, New York.

Kuznetsov, Y. A., and Rinaldi, S. 1996. Remarks on food chain dynamics, Math. Biosci. 134, 1-33.

Lingeman, R., and Van de Klashorst, G. 1992. Local and global cycles in an acarine predator-prey system: A frequency domain analysis, Exp. Appl. Acarol. 14, 201-214.

Lloyd, A. L., and May, R. M. 1999. Synchronicity, chaos and population cycles: Spatial coherence in an uncertain world, Trends Ecol. Evol. 14, 417-418.

Maly, E. J. 1969. A laboratory study of the interaction between the predatory rotifer Asplancha and Paramecium, Ecology 50, 59-73.

McCann, K., and Yodzis, P. 1995. Bifurcation structure of a three species food chain model, Theor. Popul. Biol. 48, 93-125.

Murray, J. D. 1993. "Mathematical Biology," Second corrected ed., Springer-Verlag, Berlin.

Nachman, G. 1991. A predator-prey acarine metapopulation system inhabiting greenhouse cucumbers, Biol. J. Linn. Soc. 42, 285-303.

Ranta, E., Kajtala, V., and Lundberg, P. 1999. Synchronicity in population systems: Cause and consequence mixed, Trends Ecol. Evol. 14, 400-401.

Ripa, J. 2000. Analysing the Moran effect and dispersal: Their significance and interaction in synchronous population dynamics, Oikos 89, 175-187.

Rohani, P., Lewis, T. J., Grunbaum, D., and Ruxton, G. D. 1997. Spatial self-organization in ecology: Pretty patterns or robust reality? Trends Ecol. Evol. 12, 70-74.

Rosenzweig, M. L. 1972. Stability of enriched aquatic ecosystems, Science 175, 564-565.

Rosenzweig, M. L. 1971. Paradox of enrichment: Destabilization of exploitation ecosystems in ecological time, Science 171, 385-387.

Rosenzweig, M. L., and MacArthur, R. H. 1963. Graphical representation and stability conditions of predator-prey interactions, Am. Nat. 97, 209-223.

Royama, T. 1992. "Analytical Population Dynamics," Chapman \& Hall, London.

Segel, L. A., and Jackson, J. L. 1972. Dissipative structure: An explanation and an ecological example, J. Theor. Biol. 35, 545-559.

Solé, R. V., and Gamarra, J. G. P. 1998. Chaos, dispersal and extinction in coupled ecosystems, J. Theor. Biol. 193, 539-541.

Stacey, P. B., Johnson, V. A., and Taper, M. L. 1997. Migration within metapopulations: The impact upon local population dynamics, in "Metapopulation Biology: Ecology, Genetics and Evolution" (I. Hanski and M. Gilpin, Eds.), Academic Press, San Diego.

Taylor, A. D. 1990. Metapopulations, dispersal, and predator-prey dynamics: An overview, Ecology 71, 429-433.

Van de Klashorst, G., Les Readshaw, J. L., Sabelis, M. W., and Lingeman, R. 1992. A demonstration of asynchronous local cycles in an acarine predator-prey system, Exp. Appl. Acarol. 14, 185-199.

Van der Laan, J. D., and Hogeweg, P. D. 1995. Predator-prey coevolution-interactions across different timescales, Proc. R. Soc. B 159, $35-42$.

Weisser, W. W., Jansen, V. A. A., and Hassell, M. P. 1997. The effects of a pool of dispersers on host-parasitoid systems, J. Theor. Biol. 189, 413-427.

Wiggins, S. 1990. "Introduction to Applied Non-linear Dynamical Systems and Chaos," Springer-Verlag, New York.

Winfree, A. T. 1980. "The Geometry of Biological Time," SpringerVerlag, Berlin. 